\title{
Qualitative analysis of the viability of autogenous fat grafts grafted in different environments of interstitial pressure. Preliminary results and description of a new experimental model in mini-pigs ${ }^{1}$
}

\author{
Eduardo Gustavo Pires de Arruda', Alexandre Mendonça Munhoz", Walter Matsumoto"I, Thiago \\ Uedalv $^{\text {Iv }}$ Renata de Almeida Coudry ${ }^{v}$, Rolf Gemperlivi
}

'Assistant Professor, Plastic Surgery Division, Department of Surgery, School of Medicine, Universidade de São Paulo (USP), Brazil. Conception and design of the study, acquisition and interpretation of data.

"Associate Professor, Plastic Surgery Division, Department of Surgery, School of Medicine, USP, Sao Paulo-SP, Brazil. Conception of the study, interpretation of data, manuscript writing.

IIIPlastic Surgeon, Hospital Sírio-Libanês, Sao Paulo-SP, Brazil. Acquisition of data.

IVPlastic Surgeon, Plastic Surgery Division, Department of Surgery, School of Medicine, USP, Sao Paulo-SP, Brazil. Intelectual and scientific content of the study.

'Pathologist, Hospital Sírio-Libanês, Sao Paulo-SP, Brazil. Intelectual and scientific content of the study, pathological examinations, design of the study.

${ }^{V I}$ Full Professor, Plastic Surgery Division, Department of Surgery, School of Medicine, USP, Sao Paulo-SP, Brazil. Interpretation of data.

\begin{abstract}
Purpose: To evaluate the feasibility of an experimental model of autologous fat graft (AFG) in different interstitial pressure (IP) environments.

Methods: Three mini-pigs(Minipig-BR) with age of 8 months (weight: 25-30 kg) were used. AFG were collected from the bucal fat pad, and grafted in the intramuscular pocket (biceps femoralis muscle). IP model was based on a fusiform ressection followed by primary closure "under tension". A blood pressure catheter located in the intramuscular region connected to a pressure module was applied to quantify IP.

Results: The mean operative time was 236 min (210 - $272 \mathrm{~min})$. All the AFG and muscular segments were removed successfully. Average interstitial pressure CP and $\mathrm{H}$ were 3 and 10.6 $\mathrm{mmHg}$ respectively. The AFG were biopsied for histopathological analysis 30 days after graft. Hematoxylin-eosin staining and immunohistochemical analyzes (TNF-alpha, CD31 and Perilipine with monoclonal antibodies) were employed.

Conclusion: The data show that minipigs model could be used as a recipient site for autologous fat graft techniques and allow the development of studies to explore the AFG intake and pathophysiology response.
\end{abstract}

Key words: Mammaplasty. Models, Animal. Swine. 


\section{Introduction}

Autologous fat grafting (AFG) has been widely used in aesthetic and reconstructive surgery $^{1-9}$. The possibility to transfer significant volume of fat has greatly improved the restoration of tissue defects following oncological diseased, congenital birth defects or major traumas $^{7-10}$.

In breast reconstruction field, AFG play an important part in the surgical treatment, however, is considered an unpredictable technique, with retention rates range from $20-80 \% \%^{7,11,12}$. In fact, this undesirable outcome results in multiple costly procedures, secondary procedures and sometimes unnecessary biopsies $^{5,6}$.

Despite AFG technique was first introduced by Neuber in $1893^{13}$, only more recently some authors have described technique details, expanding its availability and making it as a constant topic in many centers worldwide ${ }^{1-12}$. However, AFG is not a standard technique and clinical series demonstrate a variability of surgical procedures and results ${ }^{5,11,14,15}$.

Besides the different AFG harvesting and processing technique available, some authors advocated that large volumes of AFG can decreased tissue perfusion and less AFG intake. This aspect is due to a reduced graft-torecipient interface, which results to apoptosis and AFG necrosis ${ }^{4,9,14}$.

Although the description of the first clinical application of AFG dates back more than a century ${ }^{2,13}$, there are still controversies regarding the harvesting technique, as well as the ideal recipient site interstitial pressure (IP) in order to improve fat graft survival ${ }^{14}$. In addition, to date there are no reports that objectively evaluate the viability of AFG integration submitted to different IP in a large animal model. Thus, new experimental models of AFG would be useful to control research bias and allow further studies on reconstructive surgery.
In this manner, the aim of this study is to analyze the applicability of this new experimental model, evaluating the AFG harvesting technique and the potential influence of the recipient site IP into AFG viability.

\section{Methods}

This project was approved by the Ethics in Research of the Instituto de Ensino e Pesquisa do Hospital Sírio-Libanês (IEP$\mathrm{HSL}$ ), under registration CEUA/2014-05 and received financial sponsorship from the Regular Program of Research Assistance, FAPESP (No. 2014/19463-3). The experimental animal model was performed at the Laboratory for Research in Experimental Surgery of the IEP-HSL, in the period of January-May 2015. The present study followed the principles of international research on animals in accordance with the Federal Law (11,794 - October 8, 2008, decree number 6689 of 15 July 2009) regulating the use of experimental animal in Brazil, being the surgeries supervised by veterinary anesthetist.

All animals (Sus scropha domesticus of the miniature variant (Minipig-BR)), underwent two procedures. In the first, AFG were collected from the head (bucal fat pad (BFP)) and then grafted in the intramuscular region (biceps femoralis muscle (BFM)). In the second procedure, which usually occurred 30 days after the first one, the AFG were biopsied for histopathological analysis.

\section{Anesthetic protocol}

The animals were kept under standard laboratory conditions and surgeries were performed under general anesthesia according to the specific anesthesia protocol in animals standardized by the IEP-HSL. The animals were pre-medicated with Ketamine $(5 \mathrm{mg} / \mathrm{kg}$ ) intramuscular, midazolam $(0.5 \mathrm{mg} / \mathrm{kg})$. General anesthesia was induced via a facemask with isoflurane ( 2 vol\% inhalation) in oxygen, after 
establishing i.v. access in an ear vein. They were then oro-tracheally intubated (Mallinckrodt, blueline oral tube, I.D 6.0, Covidien plc, Dublin, Ireland). General anesthesia was maintained by volume-controlled ventilation with Isoflurane $0.5-2.0 \%$ delivered in 40-60\% oxygen in air via a ventilator (Julian anesthesia station, Dräger, Lübeck, Germany) and continuous infusion of Midazolam (0.5 mg/kg/h, (i.v.)), propofol (8 mg /

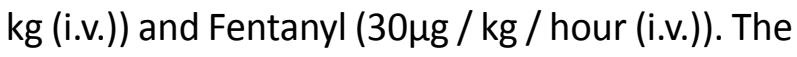
animals were kept on mechanical ventilation during the procedures and the same surgical team operated all animals.

\section{Operative procedure protocol}

\section{a) Donor area and AFG harvest}

The BFP was chosen as a donor area for the collection of the AFG. The donor and recipient areas were prepared with chlorhexidine solution and placement of sterile fields (Figure 1). Prior to AFG harvesting, local infiltration of the lateral region of the face and posterior limbs of the animals was performed with anesthetic solution $(20 \mathrm{ml}$ of $7 \%$ ropivacaine hydrochloride, 1 ampoule of adrenaline and $100 \mathrm{ml}$ of saline solution $0.9 \%$ ).

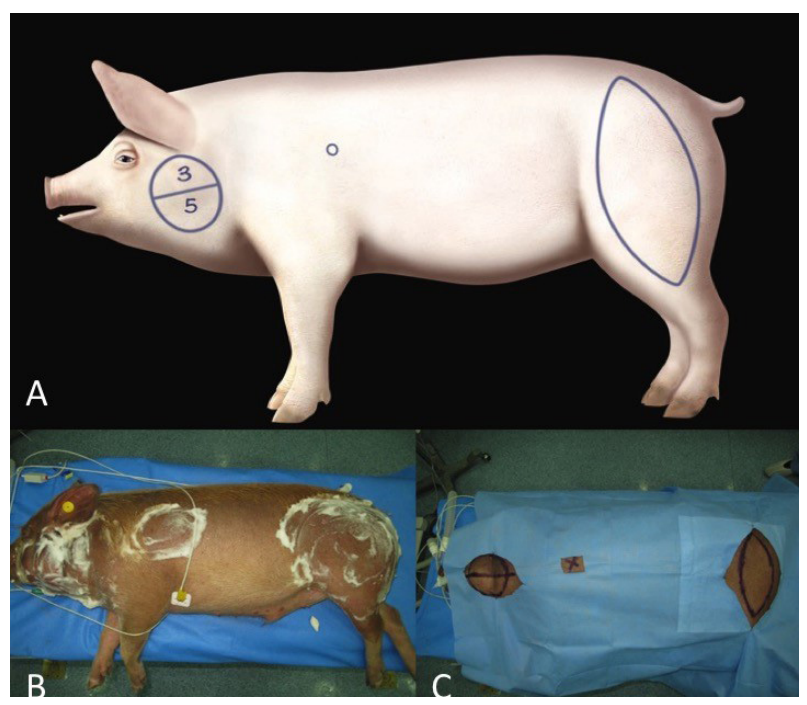

Figure 1 - Intraoperative preparation. A. Surgical site marking; B. Skin desinfection; C. Sterile surgical drape.
AFG harvesting was performed using two different techniques, conventional liposuction (LP) with cannulas co-opted in negative pressure with 3 and $5 \mathrm{~mm}$ of diameter. The upper and lower regions of the BFP were standardized in the donor area for the diameters of 3 and $5 \mathrm{~mm}$ (LP) respectively (Figure 2 ).

The AFG obtained by means of the LP was immediately centrifuged in a centrifuge for a period of 3 minutes at 3,000 revolutions per minute, with the objective of separating the AFG components (oil, fat lumps, blood and infiltration solution). The fat lumps were separated from the other tissues and then grafted with a cannula of similar size to that collected in the recipient areas (Figure $2 \mathrm{C}$ ).

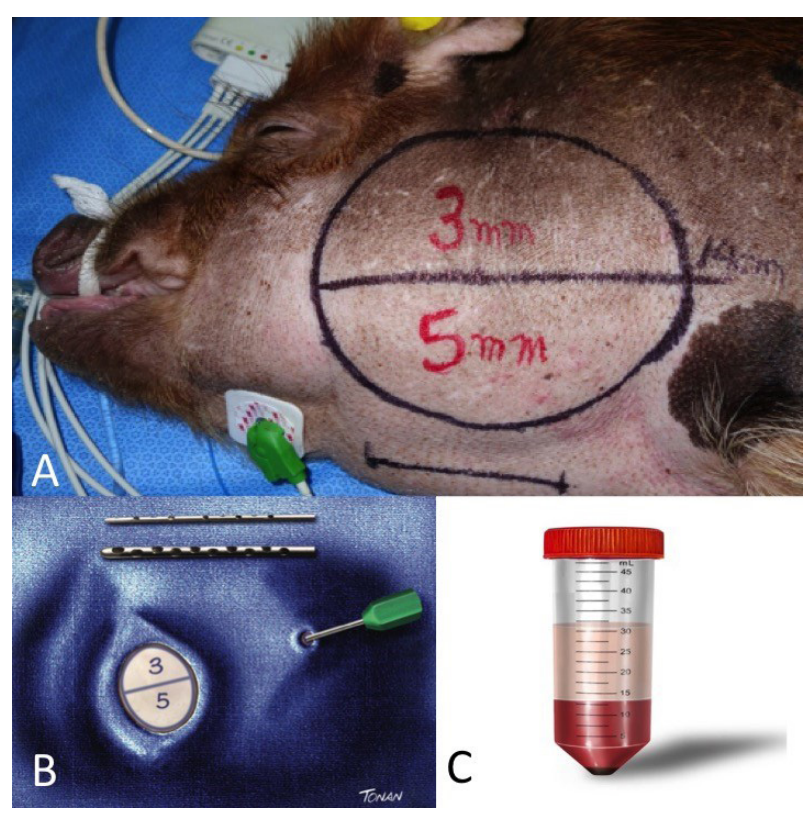

Figure 2 - A. AFG donor site; B. 3 and 5 mm cannulas; C. AFG components after centrifugation.

\section{b) AFG recipient site}

The lateral regions of the posterior limbs, right and left, were standardized as recipient sites and the femoral biceps muscle was chosen for intramuscular grafting. For this purpose, a longitudinal incision was made on the lateral side of the thigh, including the skin, the subcutaneous tissue and the superficial muscular 
fascia until identification of the muscular tissue. In order to standardize the recipient regions, eight intramuscular spaces with an approximate dimension of $0.5 \times 0.5 \mathrm{~cm}$ each were made by blunt dissection (Figure 3).

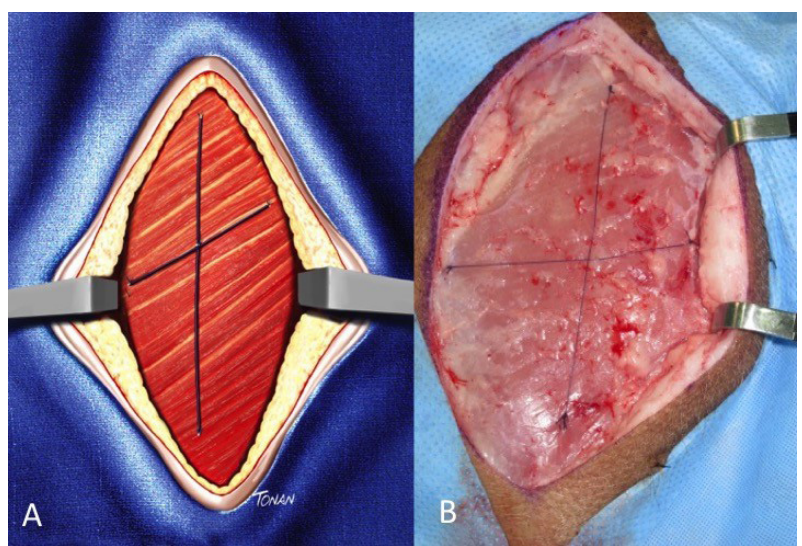

Figure 3 - A. Femoral biceps muscle scheme; B. Femoral biceps muscle dissection.

The AFG collected by LP ( 3 and $5 \mathrm{~mm}$ ) was grafted into the biceps muscle in the respective pre-determined space with cannulas of similar diameter with their respective identifications related to the respective technique (Figure 4). The muscular spaces were divided into subgroups of four and standardized for the receipt of two LP-3mm grafts and two LP- $5 \mathrm{~mm}$ grafts. In each muscular space with its respective graft (LP-3mm, LP-5mm, PD-3mm and PD-5mm), the identification was performed by means of 4-0 prolene suture stitches (Figure 5).

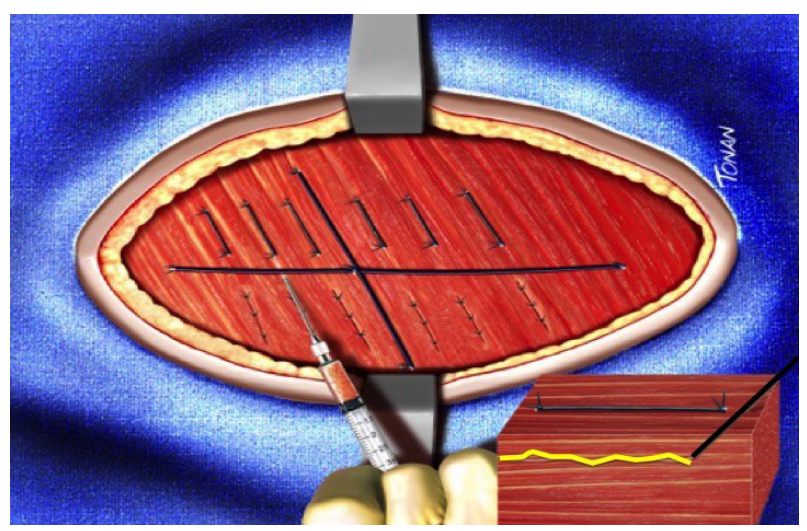

Figure 4 - Lump fat grafting.

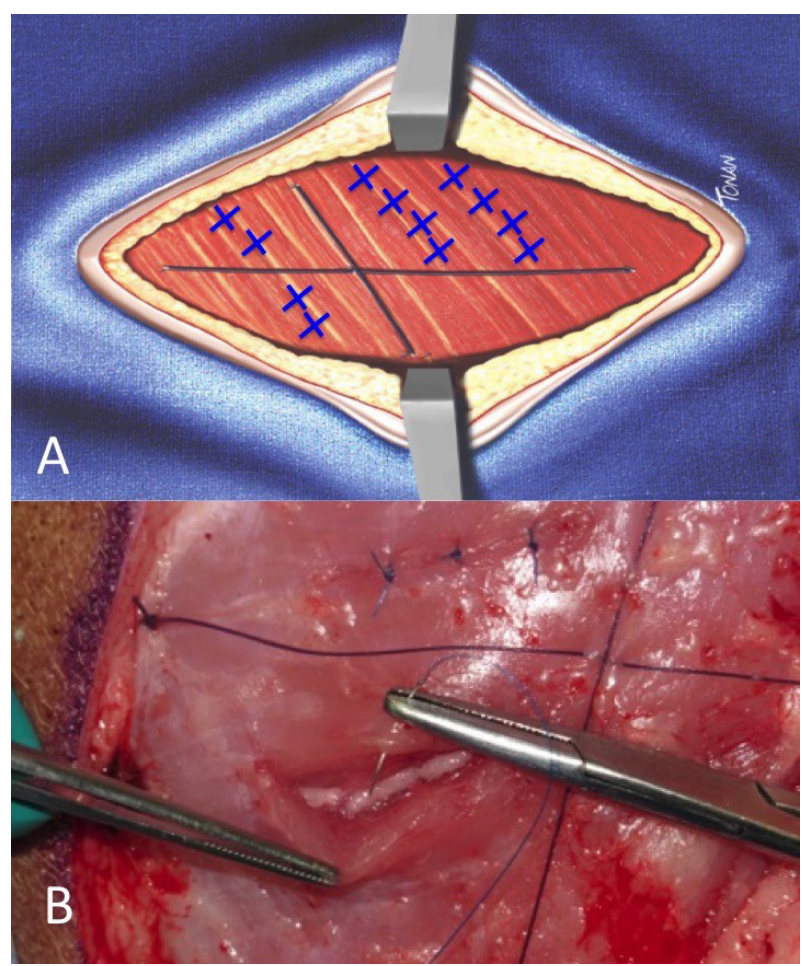

Figure 5 - A. Site demarcation; B. Closing the reception site after fat grafting.

\section{c) Interstitial pressure (IP) model}

In order to establish an interstitial pressure model, a mechanism was developed which promote and evaluate the pressure environment under physiological conditions (CF) and under a hypertension pressure (HT). For this purpose, after performing the AFT graft, the primary synthesis of the incision was performed, without tension, thus maintaining the same pressure conditions in the posterior limb (CF). In the second model (HT), a fusiform area of skin and subcutaneous tissue was resected, followed by the primary closure to promote the synthesis "under tension" and in this way favor the increase of the interstitial pressure in the lateral aspect of the posterior thigh (Figure 6).

The quantification of interstitial pressure and consequently the extent of skin/ subcutaneous resection were measured by an invasive blood pressure catheter located in the intramuscular region. This device 
was connected to an anesthesia monitor with pressure measurement module. Tissue hypertension was defined as the $20-\mathrm{mmHg}$ detected in the arterial pressure module measured by the anesthesia monitor. In the CF model, the same pressure measurement was evaluated and values close to $6-8 \mathrm{mmHg}$ were identified following the primary closure of the incision. With the aim of standardization, we defined as the left limb as HT and the right one as CF (Figure 7).

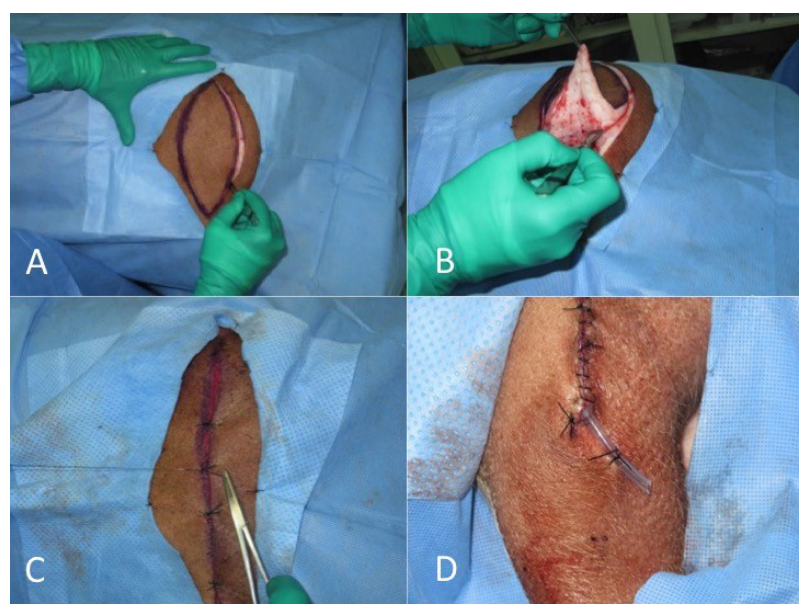

Figure 6 - A, B. Resection of a fusiform area of skin and subcutaneous tissue to achieve hypertension environment; C. Synthesis "under tension"; D. Drain

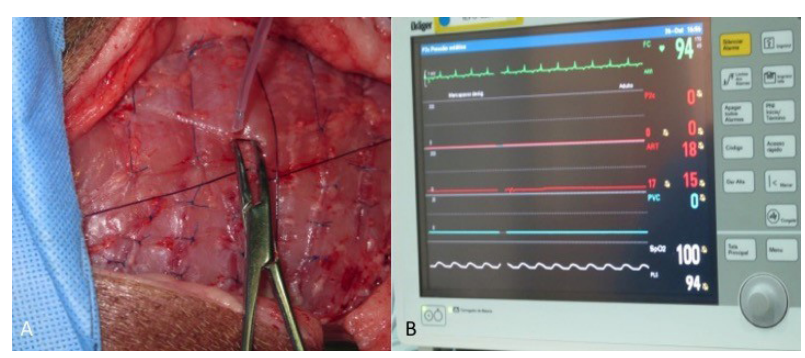

Figure 7 - A, B. Resection of a fusiform area of skin and subcutaneous tissue to achieve hypertension environment; C. Synthesis "under tension"; D. Drain.

In this way the following groups were standardized according to caliber and tissue pressure:

- Group I. AFG obtained by LP, of different calibers, placed in the intramuscular pocket of the right thigh in CF:
Group la: $3 \mathrm{~mm}$ cannula

Group Ib: $5 \mathrm{~mm}$ cannula

- Group II. AFG obtained by LP, of different calibers, placed in the intramuscular pocket of the left thigh in $\mathrm{HT}$ :

\section{Group Ila: $3 \mathrm{~mm}$ cannula}

Group IIb: $5 \mathrm{~mm}$ cannula

In the first 5 postoperative days, the animals received antibiotic prophylaxis with cefazolin (10mg / kg / day - oral) and analgesia with tramadol (2mg / kg every 12 hours - oral) and dipyrone (25mg / kg Hours - oral). During the observation period, the animals receive free supply of food and water. Each animal was kept alive for a minimum of 28 days in order to ensure integration of the grafted AFG. After this period, each animal was submitted to the second surgical procedure, respecting the same anesthetic and technical conditions of the first stage. In this second phase and with the animal in lateral decubitus, the previous scar incised and a dissection of the cutaneous, subcutaneous and superficial fascia was performed until identification of the muscular plane where the AFG were located. The biceps femoralis was partially resected bilaterally, incorporating each grafted area (4 groups per limb). Each segment was identified and filled into flasks containing $10 \%$ buffered formalin. After collecting the samples, the animal was then submitted to euthanasia (potassium chloride (66mg / kg) intravenously) (Figure 8).

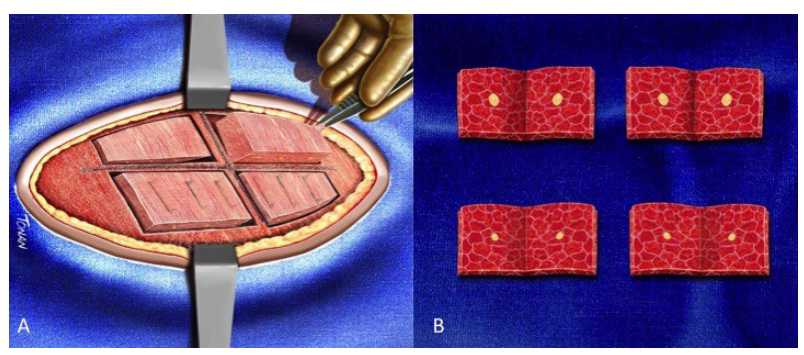

Figure 8 - A. The biceps femoralis was partially resected bilaterally, incorporating each grafted area (4 groups per limb); B. Biopsy scheme.

\section{d) Histhopathological evaluation}

The blocks containing $A F G$ and fixed 
muscle segments were processed into paraffin blocks and submitted to histological sections for specific staining. Hematoxylin-eosin staining was used for this first step. In a second step, immunohistochemical analyzes (IHC) were performed to detect the expression of TNFalpha, CD31 and Perilipine with monoclonal antibodies. IHC reactions were performed by the polymer technique in paraffin-shaped sections. The slides containing the samples were deparaffinized and antigenic recovery was performed by wet heat followed by endogenous peroxidase blockade with hydrogen peroxide $20 x$ incubated overnight with rabbit polyclonal serum $(200 \mu \mathrm{g}$ of IgG in $1 \mathrm{~mL}$ PBS with $<0.1 \%$ acid Ascorbic acid and $0.1 \%$ gelatin as the primary antibody). Subsequently, it was incubated with Envision Plus (Dako) as a peroxidase-coupled polymer for the detection and amplification of the IHC reaction. In a second slide, NovoLink (Novocastra) was used as the short detection polymer coupled to peroxidase. IHC marking was blindly quantified independently by an observer using a histological score with weights assigned to the different intensities of IHC labeling. The ratio of labeled cells will be multiplied by the intensity of the labeling to obtain a score of $0-300$, according to the previous formula. Positive controls were known to be positive tissues for the specific antibodies or lysates of transfected cells that are commercially sold for this purpose and the negative control were performed excluding the primary antibody.

\section{- TNF-alpha}

The evaluation of the presence of the marker was performed on the adipocytes present in the solid pattern graft foci and on the disseminated adipose cells infiltrating the adjacent skeletal muscle (mild, moderate and intense). The slides were then cataloged and sequenced according to: the animal, the pressure status ( $\mathrm{CF}$ and $\mathrm{HT}$ ) and cannula diameter ( 3 or $5 \mathrm{~mm}$ ).
- CD31

CD31 antigen is closely involved in the events of angiogenesis. Microvessel quantification was performed by evaluating two hotspot areas and considering the total sum of this count.

\section{- Perilipine}

Perilipine, in its isomorphic $A$, is the most abundant protein in lipid corpuscles of viable adipocytes. The differentiation between viable and non-viable adipocytes was done by perilipine analysis. The analysis was performed considering loss of expression in adipocytes.

\section{Results}

\section{General aspects}

Six surgical procedures were performed by a single surgeon on 3 domestic minipigs-BR. The animals were comparable with respect to age (8 months approximately), weight (25$32 \mathrm{~kg}$ ) and gender (all males). All procedures (AFG harvesting and biopsies) were completed without complications. The mean operative time of the first stage was 236 min (ranging 210 - $272 \mathrm{~min}$ ). All the AFG and muscular segments were removed successfully. Average interstitial pressure $\mathrm{CP}$ and $\mathrm{H}$ were 3 and $10.6 \mathrm{mmHg}$ respectively (Table 1 ). AFG obtained from 3 Mini-pigs were analyzed.

\section{Histological evaluation (HE) immunohistochemical analyzes)}

The objective of the histopathological examination was to identify the areas of intact adipocytes, fatty necrosis, fibrosis, inflammation and the presence of blood vessels. Qualitative and quantitative analyzes were performed to compare the viability of the grafted tissues in the four groups formed by the association of: cannula, diameters of $3 \mathrm{~mm}$ and $5 \mathrm{~mm}, \mathrm{CP}$ and $\mathrm{H}$ pressure. 
Table 1 - Animals data including gender, weight, intersticial pressure (maximum, average) and operative times.

\begin{tabular}{|c|c|c|c|c|c|c|c|c|}
\hline $\begin{array}{l}\text { Minipig- } \\
\text { BR }\end{array}$ & Gender & Weight & $\begin{array}{c}\text { Interstitial } \\
\text { Pressure - CP } \\
* \\
\text { (Maximum) }\end{array}$ & $\begin{array}{c}\text { Interstitial } \\
\underset{*}{\text { Pressure - CP }} \\
\text { (Average) }\end{array}$ & $\begin{array}{c}\text { Interstitial } \\
\text { Pressure - H } \\
* * \\
\text { (Maximum) }\end{array}$ & $\begin{array}{c}\text { Interstitial } \\
\text { Pressure - H } \\
* * \\
\text { (Average) }\end{array}$ & $\begin{array}{l}\text { Operative } \\
\text { Time } \\
\text { 1St. Stage }\end{array}$ & $\begin{array}{l}\text { Operative } \\
\text { Time } \\
\text { 2nd. } \\
\text { Stage }\end{array}$ \\
\hline$\# 1$ & male & $25 \mathrm{~kg}$ & $4 \times 3$ mmhg & $3 \mathrm{mmhg}$ & \multirow{3}{*}{$\begin{array}{c}11 \times 9 \\
\mathrm{mmhg} \\
14 \times 10 \\
\mathrm{mmhg} \\
11 \times 10 \\
\mathrm{mmhg}\end{array}$} & $10 \mathrm{mmhg}$ & $4 \mathrm{~h} 32 \mathrm{~min}$. & \multirow{3}{*}{$\begin{array}{c}2 \mathrm{~h} 25 \\
\min . \\
2 \mathrm{~h} 37 \\
\min . \\
1 \mathrm{~h} 59 \\
\text { min. }\end{array}$} \\
\hline \#2 & male & $32 \mathrm{~kg}$ & $4 \times 3 \mathrm{mmhg}$ & $3 \mathrm{mmhg}$ & & $12 \mathrm{mmhg}$ & $3 \mathrm{~h} 30 \mathrm{~min}$. & \\
\hline \#3 & male & $28 \mathrm{~kg}$ & $4 \times 3 \mathrm{mmhg}$ & $3 \mathrm{mmhg}$ & & $10 \mathrm{mmhg}$ & $3 \mathrm{~h} 47 \mathrm{~min}$. & \\
\hline
\end{tabular}

\section{a) Hematoxylin \& eosin staining (HE)}

The analysis was performed with focus on intramuscular grafted fat and adjacent striated muscle tissue, using $x 40$ magnification. The intact adipocytes were evaluated and assigned when present in batches or disseminated in the skeletal muscle. An average between the fields evaluated for the final score was performed. The inflammatory infiltrate, presence of multinucleated histiocytes and fatty necrosis were evaluated in a qualitative way (in mild, moderate or intense). For the analysis of these three variables an average between positive and negative fields were performed for the final score (Figure 9).

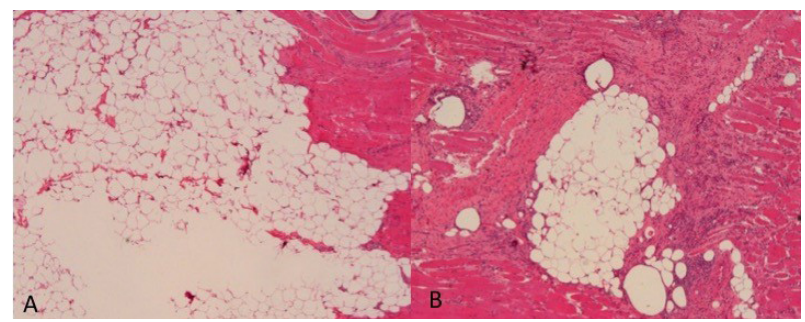

Figure 9 - Hematoxylin \& eosin staining (HE). A. Intact adipocytes; B. Inflammatory infiltrate, presence of multinucleated histiocytes and fatty necrosis.

\section{b) Immunohistochemical analyzes (IHC)} Immunohistochemical reactions for evaluation of TNF-alpha, CD31 and Perilipine proteins were performed by the polymer technique in paraffin-shaped sections. TNF alpha is a cellular signaling protein (cytokine) involved in systemic inflammation, and is the main mediator of the inflammatory lesion of the acute phase. It is produced mainly by activated macrophages and its labeling has also been described in mature adipocytes of obese patients (Figure 10). CD31 antigen is closely involved in the events of angiogenesis. Microvessel quantification was performed by evaluating two hotspot areas and considering the total sum of this count (Figure 11). Perilipine, in its isomorphic $A$, is the most abundant protein in lipid corpuscles of viable adipocytes. The differentiation between viable and non-viable adipocytes is not due to the staining of hematoxylin and eosin, but to the IHC for perilipine. The analysis was performed considering loss of expression in adipocytes (Figure 12).

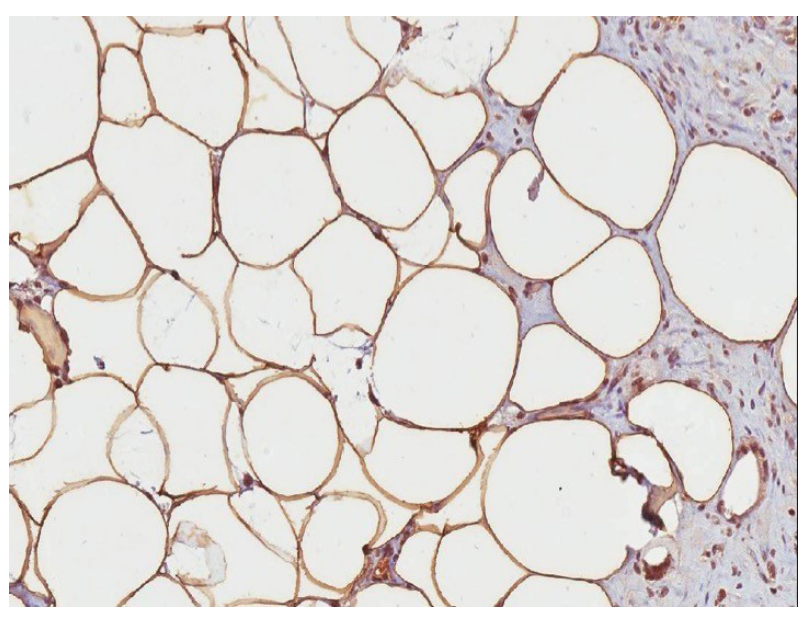

Figure 10 - Immunohistochemical staining of TNFalpha protein. Adipocytes with strong staining $(3+$ positivity). 


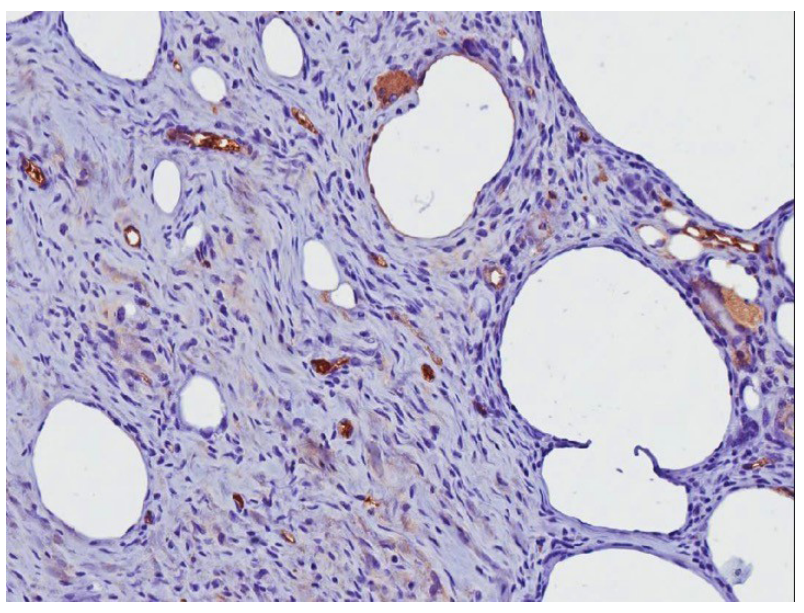

Figure 11 - Immunohistochemical staining of CD31. Microvessels and fibrosis presence.

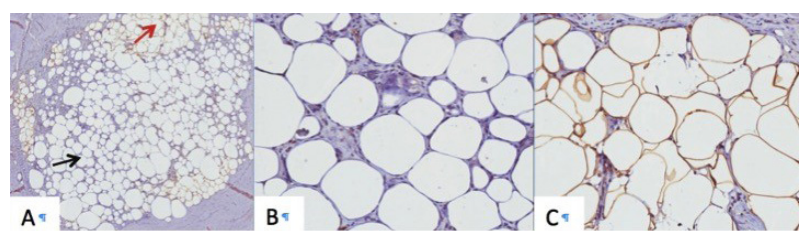

Figure 12 - Immunohistochemical staining of perilipine. A. Perilipine loss of expression (black arrow); Perilipine expression (red arrow). B. Perilipine loss of expression in detail; C. Perilipine expression in detail.

\section{Discussion}

Autologous fat grafting (AFG) has been widely used in plastic surgery and has allowed reconstruction of different types of tissue defects $^{1-12}$. According to the International Society of Aesthetic Plastic Surgery, in 2009, AFG represented almost 6 percent of the nonsurgical aesthetic procedures, with more than 500,000 procedures performed globally ${ }^{15,16}$.

In breast reconstruction field, AFG play an important part in the surgical treatment and rehabilitation ${ }^{4-6}$. The main benefits are related to low donor-site morbidity, operative time and more natural aesthetic results. However, fat grafting is considered unpredictable, with variable retention rates and resulting in local fibrosis, calcifications, oil cysts, or unsatisfactory aesthetic outcomes ${ }^{5,6,11}$. These unfavorable results in multiple costly procedures, and sometimes multiple revision procedures ${ }^{5,6}$.

Introduced in clinical practice in the last century and renewed with the studies of Illouz in the 80 ' $\mathrm{s}^{3}$, the use of AFG has been based on three steps: harvesting, processing and grafting ${ }^{5}$. Several authors have technical variations, expanding its availability and making it as a constant search topic in many centers worldwide ${ }^{5}$. However, their results demonstrate the variability of surgical procedures with current literature only supporting general principles and not any specific technique. Interestingly, there is not a standard technique available including variations on fat harvesting, preparation, and re-injection ${ }^{5,15}$.

Some authors advocated that large volumes of AFG can increase interstitial pressure leading to decreased tissue perfusion and less AFG intake ${ }^{5,14}$. In addition, the reduced graft-to-recipient interface results to apoptosis and AFG necrosis. Thus, some researches suggested an increase of the recipient site with a lower pressure environment ${ }^{14}$.

Although the description of the first clinical application of AFG dates back more than a century ${ }^{2,14}$, there are still controversies regarding the harvesting technique, the diameter of the instruments used for the aspiration as well as the recipient site pressure as a predominant factors in the long-term AFG survival ${ }^{5}$.

The domestic pig and the miniature variant (mini-pig) have been used in different areas of research and medical education ${ }^{17,18}$. The advantages are related to the similarity with human anatomy and physiology, especially with related to the skin, subcutaneous tissue skeleton, and immunological system ${ }^{18}$. Recently, the porcine models have been replacing dogs in experimental models due to ethical restrictions, and because they are more endorsed as laboratory animal models. 
In our study, the minipig-Br was advantageous since it presented an adequate donor site, the BFP, which constitutes an adequate and accessible source of AFG. The posterior limbs are a satisfactory recipient area, allowing direct perception of AFG during the grafting procedure and the possibility to evaluate two different techniques in the same animal, thus excluding variations that could affect the analysis.

In spite of these aspects, to date there are few studies that objectively evaluate the viability of integrating AFG in a large experimental model and that adequately simulate clinical practice in humans. In fact, previous animal models for AFG have the disadvantage that does not allow simulating the fat grafting process ${ }^{19-21}$. In addition, these animals' models presents fatty deposits that are disproportionately reduced when compared to liposuction cannulas normally used in humans, and therefor making it difficult to reproduce the surgical procedure. The paucity of previous studies evaluating quantification aspects of the viability of AFG, likewise the absence of any study concerning the influence of different techniques and interstitial pressure in AFG intake, has stimulate us to accomplish the present investigation.

Previous studies using small animals have evaluated the viability of AFG and suggested different technical aspects to obtain better integration ${ }^{19,20,22}$. Karacaoglu et al. ${ }^{20}$ analyzed the AFG volume maintenance in different recipient sites in a rabbit model. Based on histopathologic analyses, the authors observed a significant increase of fat graft survival in supramuscular layer (81.95\%) than in submuscular layer (37.31\%) $(\mathrm{P}<0.05)$. Contrary, Guerrerosantos et al. in a rat model noted that there are lower absorption rates when the fat is injected into muscle ${ }^{22}$.

Other authors analyzed the influence of the environment in terms of AFG intake ${ }^{23,24}$. Lee et al. analyzed AFG recipient sites of
New Zealand rabbits and the role of negative pressure to improve the vascularity and fat survival ${ }^{23}$. According to the authors, AFG survival rate and skin perfusion was higher in the negative pressure group (75.4\%) than in the control group $(53.1 \%)(P<0.001)$. Findlay et al. using a porcine model evaluated the role of tissue engineering in replacement normal tissues ${ }^{24}$. For this purpose, large-volume chambers were implanted enclosing a fat flap and compared with chambers included sponge in association with muscle or AFG.

Concerning the AFG harvesting technique, the methods used varied widely. Most clinical studies used manual procedure with a 2 to $5 \mathrm{~mm}$ cannula attached to a 2-60cc syringe ${ }^{5}$. In spite of the controversy related to cannula diameters, the technique of grafting significantly affects the rate of AFG absorption $^{5,11}$. In fact, when large volumes of AFG are grafted a raise in cyst formation, infection, fibrosis and absorption may occur ${ }^{5}$. On the other hand, grafting small volumes of AFG potentially increases the surface area between the graft and recipient site, and improves integration ${ }^{11}$. In our study, the AFG was collected by cannulas with 3 and $5 \mathrm{~mm}$ of diameter and were grafted into the biceps muscle with cannulas of similar diameter. It has been our clinical experience that AFG intake is dependent on diffusion of nutrients from adjacent tissues and, consequently, fat particles has to be kept small to improve viability. Carpaneda et al. ${ }^{25}$ studied AFG viability as a function of grafted volume and hypothesized that AFG nutrition is achieved by imbibition over a distance of $1.5 \mathrm{~mm}$ from the edge of the fat. In fact, smaller grafts have an advantage over larger ones, as a greater proportion of the graft is in direct contact with the recipient tissue, which facilitates the process of revascularisation ${ }^{11}$.

Similar concept can be applied to the recipient site interstitial pressure and its 
relationship to AFG integration. In fact, some studies demonstrated that AFG has a low tolerance to ischemia ${ }^{14,19}$. From the physiologic point of view, large volumes of AFG can increase interstitial pressure leading to decreased tissue perfusion and less AFG intake ${ }^{4,14}$. In addition, the reduced graft-to-recipient interface results to apoptosis and necrosis of the AFG. Based on this concept and the influence of interstitial pressure, some authors advocates an increase of the parenchymal space and the creation of lower pressure environment ${ }^{4,14}$.

In order to quantify the AFG viability we utilized histological sections for specific staining. Hematoxylin-eosin staining and immunohistochemical (IHC) analyzes to detect the expression of TNF-alpha, CD31 and Perilipine with monoclonal antibodies. For this purpose, a histological score was used with weights assigned to the different intensities of IHC labeling. TNFa (Tumor Necrosis Factor alpha) gene encodes a multifunctional proinflammatory cytokine and is produced by a large variety of cells, including macrophages ${ }^{26}$. This cytokine is related in the regulation of a wide spectrum of biological processes including cell proliferation, apoptosis, and lipid metabolism. To assess tissue vascularity, sections were immunohistochemically stained for CD31. This molecule is a single chain type1 transmembrane protein that participate in adhesive interactions between adjacent endothelial cells and is the most sensitive and specific marker of endothelial differentiation ${ }^{27}$. The CD31 also known as PECAM-1 (Platelet Endothelial Cell Adhesion Molecule-1) has been largely used in microvessel density assessment in different fields of research, including primary melanoma, tumor angiogenesis, wound healing and fat grafting integration ${ }^{28}$. Finally, Perilipin also known as lipid droplet-associated protein, is a protein associate with the surface of lipid droplets. This protein is an important regulator of lipid storage, and its phosphorylation is important for the mobilization of fats in adipose tissue ${ }^{29}$. In research field, it has been demonstrated that dead adipocytes are negative for perilipin ${ }^{30}$. In our study, dead adipocytes were not differentiated from living adipocytes in conventional stain such as Hematoxylin and Eosin. Thus, the use of Perilipin allowed differentiation between dead adipocytes (perilipin-negative from living adipocytes (perilipin-positive) in immunostaining.

The swine model has been regularly applied for experimental research, as well as for surgical investigations due to its correspondence to humans in terms of anatomy and physiology. The strengths of the present model include the use of a new experimental model to evaluate the feasibility of the AFG in vivo and under different scenarios of pressure, which has not been reported in previous studies. On the other hand, limitations are the small number of animals analyzed. Because the present pilot study assure that the experimental model on $A F G$ is viable, we are now conducting experiments with a larger sample to investigate the role of different harvesting techniques as well as the influence of potential tissue factors into the process of AFG intake. Thus, these experimental studies of AFG in progress would be useful to control research bias and allow further researches on reconstructive surgery.

\section{Conclusions}

Autologous fat grafting is increasingly used for soft tissue reconstruction due to availability, biocompatibility and low donor-site morbidity. However, the technique is generally considered an unpredictable procedure. The miniature pig variant (mini-pig) have been used in different areas of research and the advantages are related to the similarity with human anatomy and physiology. In our study, the experimental model presented an adequate donor site, and a satisfactory recipient area, allowing direct perception of AFG during the grafting procedure 
and the possibility to evaluate different techniques in the same animal. The present data show that the porcine model can be used as a recipient site for AFG techniques. This new experimental model will allow the development of studies to explore the intake of AFG and initial pathophysiology response.

\section{References}

1. Chajchir A. Fat injection: long-term followup. Aesthetic Plast Surg. 1996;20:291-6. PMID: 8791566.

2. Billings Jr E, May Jr JW. Historical review and present status of free fat graft autotransplantation in plastic and reconstructive surgery. Plast Reconstr Surg. 1989;83:368-81. PMID: 2643129.

3. Illouz YG. Body contouring by lipolysis: a 5-year experience with over 3000 cases. Plast Reconstr Surg. 1983;72:591-7. PMID: 6622564.

4. Khouri R, Del Vecchio D. Breast reconstruction and augmentation using pre-expansion and autologous fat transplantation. Clin Plast Surg. 2009;36:269-80. PMID: 19309653.

5. Gir P, Brown SA, Oni G, Kashefi N, Mojallal A, Rohrich RJ. Fat grafting: evidence-based review on autologous fat harvesting, processing, reinjection, and storage. Plast Reconstr Surg. 2012;130:249-58. PMID: 22743888.

6. Delay E, Garson S, Tousson G, Sinna R. Fat injection to the breast: technique, results, and indications based on 880 procedures over 10 years. Aesthet Surg J. 2009;29:36076. PMID: 19825464.

7. Del Vecchio D, Rohrich RJ. A classification of clinical fat grafting: different problems, different solutions. Plast Reconstr Surg. 2012;130:511-22. PMID: 22929236.

8. Coleman SR, Saboeiro AP. Fat grafting to the breast revisited: safety and efficacy. Plast Reconstr Surg. 2007;119:775-85. PMID: 17312477.

9. Sampaio Goes JC, Munhoz AM, Gemperli R. The subfascial approach to primary and secondary breast augmentation with autologous fat grafting and form-stable implants. Clin Plast Surg. 2015;42(4):551-64. PMID: 26408443.

10.Munhoz AM, Montag E, Filassi JR, Gemperli R. Immediate nipple-areola-sparing mastectomy reconstruction: An update on oncological and reconstruction techniques. World J Clin Oncol. 2014 10;5(3):478-94. PMID: 25114861.

11.Gause TM, Kling RE, Sivak WN, Marra KG, Rubin JP, Kokai LE. Particle size in fat graft retention: A review on the impact of harvesting technique in lipofilling surgical outcomes. Adipocyte. 2014 10;3(4):273-9. PMID: 26317051.

12.Zocchi M, Zuliani F. Bicompartmental breast lipo-structuring. Aesthet Plast Surg. 2008;32:313-28. PMID: 18188638.

13.Arcuri F, Brucoli M, Baragiotta N, Stellin L, Giarda M, Benech A. The role of fat grafting in the treatment of posttraumatic maxillofacial deformities. Craniomaxillofac Trauma Reconstr. 2013 Jun;6(2):121-6. PMID: 24436747.

14.Khouri RK Jr, Khouri RE, Lujan-Hernandez JR, Khouri KR, Lancerotto L, Orgill DP. Diffusion and perfusion: the keys to fat grafting. Plast Reconstr Surg Glob Open. 2014 7;2(9):e220. PMID: 25426403.

15.Gutowski K; ASPS Fat Graft Task Force. Current application and safety of autologous fat grafts: A report of the ASPS fat graft task force. Plast Reconstr Surg. 2009;124:272-80. PMID: 19346997.

16.American Society of Plastic Surgeons. Fat transfer/fat graft and fat injection: ASPS guiding principles. Available from http:// www.plasticsurgery.org/Documents/ medical-professionals/ health-policy/ guiding-principles/ASPS-Fat-Transfer-GraftGuiding-Principles.pdf.

17.Dantzer R. The pig as a model for behavioral research. Lab An Sci. 1986;36:362-5. PMID: 3534439.

18.Swindle MM, Smith AC, Laber-Laird K, Dungan L. Swine in biomedical research: management and models. ILAR J. 1994;36:15. PMID: 3154081.

19.Fraga MF, Helene A Jr, Nakamura F, Lellis RF, 
Kikuchi W, Esteban D. Comparative study of the integration and viability of autonomised and nonautonomised autologous fat tissue grafts: experimental model in rabbits. J Plast Reconstr Aesthet Surg. 2008;61(9):1044-8. PMID: 17884744.

20.Karacaoglu E, Kizilkaya E, Cermik H, Zienowicz $R$. The role of recipient sites in fat-graft survival: experimental study. Ann Plast Surg. 2005;55:63-8. PMID: 15985793.

21.Jung DW, Kim YH, Kim TG, Lee JH, Chung KJ, Lim JO, Choi JY. Improvement of fat transplantation: fat graft with adipose-derived stem cells and oxygengenerating microspheres. Ann Plast Surg. 2015;75(4):463-70. PMID: 26207545.

22.Guerrerosantos J, Gonzalez-Mendoza A, Masmela Y, Gonzalez MA, Deos M, Diaz P. Long-term survival of free fat grafts in muscle: an experimental study in rats. Aesthetic Plast Surg. 1996;20(5): 403-8. PMID: 8849432.

23.Lee JW, Han YS, Kim SR, Kim HK, Kim H, Park $\mathrm{JH}$. A rabbit model of fat graft recipient site preconditioning using external negative pressure. Arch Plast Surg. 2015;42(2):150-8. PMID: 25798385.

24.Findlay $\mathrm{MW}$, Dolderer $\mathrm{JH}$, Trost $\mathrm{N}$, Craft RO, Cao Y, Cooper-White J, Stevens G, Morrison WA. Tissue-engineered breast reconstruction: bridging the gap toward large-volume tissue engineering in humans. Plast Reconstr Surg. 2011;128(6):1206-15.
PMID: 22094739.

25.Carpaneda CA, Ribeiro MT. Percentage of graft viability versus injected volume in adipose autotransplants. Aesthetic Plast Surg. 1994;18:17-9. PMID: 8122571.

26.Idriss HT, Naismith JH. TNF alpha and the TNF receptor superfamily: structure-function relationship(s). Microsc Res Tech. 2000 1;50(3):184-95. PMID: 10891884.

27.Parums DV, Cordell JL, Micklem K, Heryet AR, Gatter KC, Mason DY. A new monoclonal antibody that detects vascular endothelium associated antigen on routinely processed tissue sections. J Clin Pathol. 1990;43(9):7527. PMID: 2212067.

28.DeLisser HM, Christofidou-Solomidou M, Strieter RM, Burdick MD, Robinson CS, Wexler RS, Kerr JS, Garlanda C, Merwin JR, Madri JA, Albelda SM. Involvement of endothelial PECAM-1/CD31 in angiogenesis. Am J Pathol. 1997;151(3):671-7. PMID: 9284815.

29.Tansey JT, Sztalryd C, Hlavin EM, Kimmel $A R$, Londos $C$. The central role of perilipin an in lipid metabolism and adipocyte lipolysis. IUBMB Life. 2004;56:379-85. PMID: 15545214.

30.Eto $H$, Kato $H$, Suga $H$, Aoi $N$, Doi $K$, Kuno $S$, Yoshimura K. The fate of adipocytes after nonvascularized fat grafting: evidence of early death and replacement of adipocytes. Plast Reconstr Surg. 2012;129(5):1081-92. PMID: 22261562.

\section{Correspondence:}

Alexandre Mendonça Munhoz

Instituto de Ensino e Pesquisa, Hospital SírioLibanês

Rua Mato Grosso, 306 cj.1705-1706

01239-040 São Paulo - SP Brasil

munhozalex@uol.com.br

Received: July 27, 2017

Review: Sept 28, 2017

Accepted: Oct 30, 2017
Conflict of interest: none

Financial source: FAPESP (no 2014/19463-3) 